\title{
Microwave-Assisted Acid Digestion: Evaluation of Reaction Vessel Design and Performance
}

\author{
Julia A. Garitta, ${ }^{a}$ Lucimar L. Fialho, ${ }^{a}$ Glenda S. de Oliveira, ${ }^{a}$ Roberta M. Maria, ${ }^{\oplus a}$ \\ Camillo Pirola, ${ }^{b}$ Antônio G. Ferreira ${ }^{a}$ and Joaquim A. Nóbrega ${ }^{\circledR *, a}$ \\ ${ }^{a}$ Departamento de Química, Universidade Federal de São Carlos, \\ CP 676, 13560-270 São Carlos-SP, Brazil
}

${ }^{b}$ Milestone Srl, 24010 Sorisole-BG, Italy

\begin{abstract}
Nowadays, microwave-assisted procedures using closed vessels with thermal, chemical, and mechanical resistance are the state-of-the-art for efficient digestion of samples. Safety issues related to sample reactivity should be considered and analytical throughput is also a critical parameter. The choice of a specific vessel for a target application is not trivial and simple experiments are presented here for rice flour and bovine liver samples to illustrate effects of vessel design on digestion performance. Despite using the same heating program, the residual carbon contents varied from 22 to $67 \%$ to bovine liver digests and from 7 to $96 \%$ to rice flour digests. Quantitative recoveries were obtained for most analytes. Low recoveries were observed mainly for $\mathrm{Ca}$ and $\mathrm{Fe}$. Analytical performance is related to different sizes, shapes and the gradient of temperature for each model of digestion vessel. It was demonstrated that taller vessels improved regeneration of nitric acid.
\end{abstract}

Keywords: microwave radiation, sample preparation, closed vessel, digestion efficiency, ICP OES

\section{Introduction}

Kingston and Jassie ${ }^{1}$ and Kingston and Haswell ${ }^{2}$ have published landmark books that demonstrated the benefits of microwave radiation for sample digestion and for enhancing chemical processes in several areas of science. More recently, Krug and Rocha ${ }^{3}$ and Flores ${ }^{4}$ have edited books that demonstrated how this technology has evolved and nowadays it is broadly applied. In this context, microwaveassisted digestion has progressively become a standard procedure for sample preparation and it has clear impact in procedures applied for trace analysis and green chemistry. ${ }^{5}$

As typical in analytical chemistry, there is a feedback process between the evolution of instrumentation and the understanding of thermochemical processes allowing the development of sample digestion procedures compatible with modern analytical instruments for simultaneous determination of several analytes. Gradually, it became clear that microwave radiation is not only an alternative for fast heating of solutions containing ions and dipoles, but it is a strategy for implementing better and more effective analytical procedures. The use of dilute nitric acid solutions for performing digestions represents a step forward in the

*e-mail: djan@ufscar.br arsenal of strategies combining trace analysis and green chemistry requirements. ${ }^{5}$

This evolution relied on the design of proper microwave ovens with temperature and pressure sensors, hardware and software that supported the development of reliable, safe, and efficient analytical procedures for sample digestion. Another critical development was the design of proper reaction vessels that can withstand high pressure, high temperature and critical chemical reagents. Of course, this evolution had occurred step by step and new materials and designs were carefully evaluated. ${ }^{1-4}$ Nowadays, we have available vessels and rotors that can accommodate these critical working conditions.

In this sense, one important feature is how to choose a reaction vessel for performing digestions. One clear aspect is the relation between the maximum mass of sample and the maximum pressure a vessel can withstand. Generally, it is assumed that typical maximum masses for organic samples are around $0.5 \mathrm{~g}$ and for inorganic samples are around $1 \mathrm{~g}$ because the former generate higher volume of gases. Another critical parameter is the maximum temperature that can be applied that is related to the efficiency of digestion and vessel material. Another important point, despite being less considered, is the dimensions of the vessels. Volume is important, but two vessels may have the same volume and 
they will have different performances if their diameters and heights are different. Taller vessels have higher gradient of temperature among solution phase, gas phase and vessel walls. This will improve the regeneration of nitric acid by condensation processes. ${ }^{6}$ The use of hydrogen peroxide in the acid mixture for promoting sample digestion releases oxygen and energy to the system providing a more efficient digestion. When dilute nitric acid solution promotes the oxidation of the organic matrix of the sample, NO is formed and this compound is easily oxidized to $\mathrm{NO}_{2}$. Further processes led to regeneration of nitric acid as shown in equations below:

$$
\begin{aligned}
& 2 \mathrm{H}_{2} \mathrm{O}_{2} \rightarrow 2 \mathrm{H}_{2} \mathrm{O}+\mathrm{O}_{2(\mathrm{~g})} \\
& \left(\mathrm{CH}_{2}\right)_{\mathrm{n}}+2 \mathrm{HNO}_{3(\text { aq })} \rightarrow \mathrm{CO}_{2(\mathrm{~g})}+2 \mathrm{NO}_{(\mathrm{g})}+2 \mathrm{H}_{2} \mathrm{O}_{(\mathrm{l})} \\
& 2 \mathrm{NO}_{(\mathrm{g})}+\mathrm{O}_{2(\mathrm{~g}) \rightarrow} 2 \mathrm{NO}_{2(\mathrm{~g})} \\
& 2 \mathrm{NO}_{2(\mathrm{~g})}+\mathrm{H}_{2} \mathrm{O}_{(\mathrm{l})} \rightarrow \mathrm{HNO}_{3(\text { (aq })}+\mathrm{HNO}_{2(\mathrm{aq})}
\end{aligned}
$$

These processes were demonstrated for microwaveassisted $^{6}$ and also for conventional heating. ${ }^{7}$

This study evaluated three vessel designs for digestions of rice flour and bovine liver samples using dilute nitric acid solutions. Two types of vessels were designed for conventional microwave ovens and one type of vessel was designed for the single reaction chamber technology (SRC). This is a different type of microwave oven with all vessels inserted in a nitrogen pressurized $1 \mathrm{~L}$ chamber containing a small volume of water-acid base load that absorbs microwave radiation and transfers the heat to the partially immersed reaction vessels. The SRC technology was discussed in detail in a recent book. ${ }^{8}$

\section{Experimental}

Instruments

Two microwave ovens produced by Milestone (Milestone, Sorisole, BG, Italy) were used in this study. The first is the model ETHOS UP ${ }^{\mathrm{TM}}$ associated with either a medium pressure rotor with 44 TFM vessels (MAXI 44) or with a high-pressure rotor featuring up to 15 TFM vessels (SK-15). Figure 1 shows a picture of these vessels. The former allows maximum temperature of $200{ }^{\circ} \mathrm{C}$ and the pressure must be kept below 35 bar. On the other hand, the latter can support temperatures of up to $230^{\circ} \mathrm{C}$ and maximum working pressure of $100 \mathrm{bar}$. This oven has $1.8 \mathrm{~kW}$ microwave power source. These vessels have a volume of $100 \mathrm{~mL}$ and these rotors were controlled by contact-less infrared sensor (T2) for temperature control of all vessels.

The second microwave oven used is the UltraWAVE ${ }^{\mathrm{TM}}$ that is based on the SRC technology which overcomes

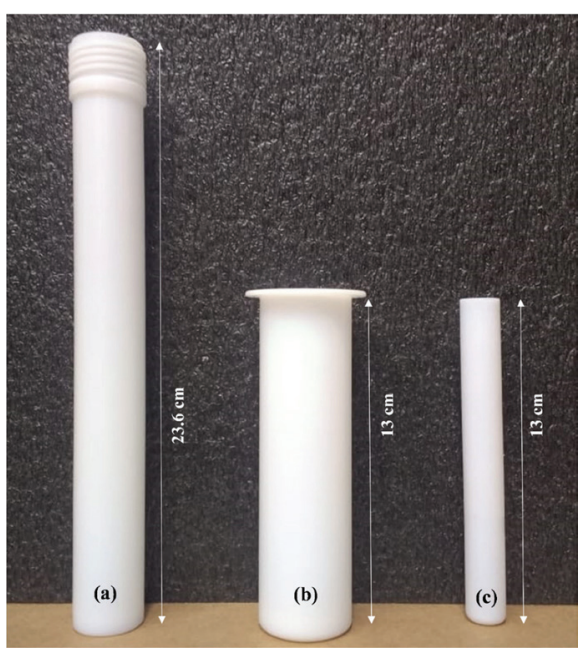

Figure 1. Digestion vessels: (a) MAXI 44, (b) SK-15 and (c) SRC.

limitations of all conventional microwave ovens. The UltraWAVE ${ }^{\mathrm{TM}}$ has a $1.5 \mathrm{~kW}$ microwave power source that delivers microwave energy to a $1 \mathrm{~L}$ chamber that usually contains $150 \mathrm{~mL}$ of deionized water plus $5 \mathrm{~mL}$ of concentrated $\mathrm{HNO}_{3}$. The racks with TFM, quartz or glass vessels stay inside this SRC. Direct pressure and temperature control of the SRC allows direct control of each sample. The UltraWAVE ${ }^{\mathrm{TM}}$ can be operated up to $199 \mathrm{bar}$ of pressure and at temperatures as high as $300^{\circ} \mathrm{C}$. Digestions were carried out using a rack with 15 TFM vessels. Each vessel has a volume of $15 \mathrm{~mL}$.

The determination of selected analytes was performed by inductively coupled plasma optical emission spectrometry (ICP OES iCAP 6000 Duo, Thermo Fisher Scientific, Madison, WI, USA) in axial viewing mode. Instrument operational conditions are shown in Table 1.

The total carbon in bovine liver and rice flour samples

\begin{tabular}{|c|c|}
\hline Instrument parameter & Operational condition \\
\hline Radiofrequency applied power $/ \mathrm{kW}$ & 1.15 \\
\hline Plasma gas flow rate / $\left(\mathrm{L} \mathrm{min}^{-1}\right)$ & 12 \\
\hline Auxiliary gas flow rate / $\left(\mathrm{L} \mathrm{min}^{-1}\right)$ & 1.5 \\
\hline Nebulizer gas flow rate $/\left(\mathrm{L} \mathrm{min}^{-1}\right)$ & 0.50 \\
\hline Sample flow rate $/\left(\mathrm{mL} \mathrm{min}^{-1}\right)$ & 1.0 \\
\hline Stabilization time / s & 5 \\
\hline Observation mode & axial \\
\hline Spectral line / $\mathrm{nm}$ & $\begin{array}{c}\text { C I } 193.091 ; \text { Ca I } 422.673 \\
\text { Ca II } 317.933 ; \text { Cu I } 324.754 \\
\text { Fe II } 238.204 ; \text { K I } 766.490 \\
\text { K I } 404.414 ; \text { Mg II } 280.270 \\
\text { Mn II } 259.373 ; \text { Na I } 588.995 \\
\text { Na I } 818.326 ; \text { P I } 177.495 \\
\text { Zn II } 202.548\end{array}$ \\
\hline
\end{tabular}
was determined by element analyzer CHNS-O (EA 1108,

Table 1. Operational conditions for inductively coupled plasma optical emission spectrometry 
Fisons Instruments, Cornaredo, MI, Italy) and the dissolved organic carbon after digestion was determined by ICP OES.

${ }^{1} \mathrm{H}$ nuclear magnetic resonance (NMR) spectra were acquired in a Bruker (Karlsruhe, Germany) equipment, AVANCE III model, $14.1 \mathrm{~T}(600.23 \mathrm{MHz}$ for the ${ }^{1} \mathrm{H}$ frequency) equipped with a $5 \mathrm{~mm}$ TXI cryogenic probe and keeping the temperature at $298 \mathrm{~K}$ during all measurements. The spectra were collected using a pulse sequence with water signal suppression performed by an excitation sculpting sequence, with 128 scans (NS), 4 dummy scans (DS), $64 \mathrm{~K}$ data points (TD), spectral window of $16 \mathrm{ppm}(\mathrm{SW})$, acquisition time (AQ) of $3.40 \mathrm{~s}$, relaxation delay $3.0 \mathrm{~s}$ (D1) and the receiver gain (RG) used was the same for all the experiments. All spectra were referenced to the sodium 3-trimethylsilyl2,2,3,3-propianate- $d_{4}$ (TMSP- $d_{4}, \delta=0.00 \mathrm{ppm}$ ) and processed with $32 \mathrm{~K}$ points (SI), using an exponential multiplication $(\mathrm{lb}=0.3 \mathrm{~Hz})$ and automatic phase and baseline correction.

Materials, samples and solutions

Distilled-deionized water was produced in a Milli- $\mathrm{Q}^{\circledR}$ System (Bedford, MA, USA) and used to prepare all solutions. Nitric acid (Labsynth, Diadema, SP, Brazil) was purified using the DuoPur apparatus (Milestone, Sorisole, Italy). Concentrated nitric acid purified by sub-boiling distillation was diluted to 3.0, 1.4, 0.7 and $0.35 \mathrm{~mol} \mathrm{~L}^{-1}$. These diluted solutions were used for sample digestions. Concentrated $\mathrm{H}_{2} \mathrm{O}_{2}\left(30 \% \mathrm{~m} \mathrm{~m}^{-1}\right)$ from Labsynth, Diadema, SP, Brazil, was also used. Reference analytical solutions were prepared in $1 \% \mathrm{v} \mathrm{v}^{-1} \mathrm{HNO}_{3}$ by dilution of stock solutions containing $1000 \mathrm{mg} \mathrm{L}^{-1}$ of each target analyte (Qhemis, São Paulo, SP, Brazil and SpecSol, Jacareí, SP, Brazil). All materials and glassware were immersed in $10 \% \mathrm{v} \mathrm{v}^{-1} \mathrm{HNO}_{3}$ for $24 \mathrm{~h}$ and rinsed with deionized water before use.

This study was performed using two reference materials: rice flour (CRM-Agro C1001a) and bovine liver (RM-AgroE3001a). The first was produced by the Centro de Energia Nuclear na Agricultura-Universidade de São Paulo (CENA-USP, Piracicaba, SP, Brazil) and the second by EMBRAPA Pecuária Sudeste (São Carlos, SP, Brazil) as part of an inter laboratory program. All digests were diluted to $50 \mathrm{~mL}$ before determination of analytes.

\section{Procedure}

Masses of approximately $500 \mathrm{mg}$ of samples were accurately weighed and transferred to $15-\mathrm{mL}$ TFM vessels and $10.0 \mathrm{~mL}$ of $\mathrm{HNO}_{3}$ solutions (3.0, 1.4, 0.7 and $\left.0.35 \mathrm{~mol} \mathrm{~L}^{-1}\right)$ or $8.0 \mathrm{~mL}$ of $\mathrm{HNO}_{3}$ solutions $(3.0,1.4,0.7$ and

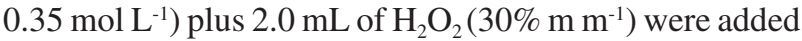
for performing digestions. Analytical blanks of different digest solutions were simultaneously prepared.

The heating program adopted in all experiments is described in Table 2. Table 3 describes the second heating program applied only in some experiments using UltraWAVE ${ }^{\mathrm{TM}}$

Table 2. Heating program adopted in all experiments when evaluating the three different vessel configurations at maximum temperature of $200{ }^{\circ} \mathrm{C}$

\begin{tabular}{lcc}
\hline Temperature $/{ }^{\circ} \mathrm{C}$ & time of ramp $/$ min & time of plateau / min \\
\hline 100 & 5 & 5 \\
200 & 5 & 15 \\
\hline
\end{tabular}

Table 3. Heating program adopted in UltraWAVE ${ }^{\mathrm{TM}}$ at higher temperature

\begin{tabular}{lcc}
\hline Temperature $/{ }^{\circ} \mathrm{C}$ & time of ramp $/$ min & time of plateau / min \\
\hline 150 & 10 & 2 \\
240 & 15 & 10 \\
\hline
\end{tabular}

The residual acidity was determined by acid-base titrations of the digests. Titrations were carried out with $\mathrm{NaOH}$ standardized solution $\left(0.0463 \mathrm{~mol} \mathrm{~L}^{-1}\right)$ and phenolphthalein as indicator. Dissolved organic carbon contents (DOC) were determined by ICP OES using solutions containing oxalic acid (Mallinckrodt, Phillipsburg, NJ, USA) for calibration. The total organic carbon (TOC) was determined using analytical curve of the acetanilide $\left(\mathrm{CH}_{3} \mathrm{CONHC}_{6} \mathrm{H}_{5}\right)$. The residual carbon content (RCC) was calculated as indicated in literature. ${ }^{4}$

The adopted sample preparation for NMR measurements was the simple addition of $100 \mu \mathrm{L}$ of deuterium oxide solution with TMSP- $d_{4}\left(0.2 \% \mathrm{v} \mathrm{v}^{-1}\right)$ as internal standard to $400 \mu \mathrm{L}$ of the sample resulted from the different digestion processes. After homogenization using a vortex type stirrer for $10 \mathrm{~min}$ the final solution was transferred to a $5 \mathrm{~mm}$ NMR tube.

\section{Results and Discussion}

This section was structured to describe the analytical performance of each rotor and respective vessels starting from the simplest one, MAXI 44, followed by SK-15 vessels, and, finally, for the SRC technology.

\section{Analytical performance: MAXI 44}

The digests of bovine liver and rice flour samples obtained using the MAXI 44 rotor with addition of $\mathrm{H}_{2} \mathrm{O}_{2}$ had better visual aspects for all tested concentrations of 
$\mathrm{HNO}_{3}$ in comparison to the digests without this reagent (Figure 2). Recoveries were quantitative for most elements in all conditions, except for $\mathrm{Ca}$ in bovine liver digests and Fe in rice flour digests (Tables 4 and 5). It was demonstrated by Wieteska et al. ${ }^{9}$ that the presence of $\mathrm{Si}$ in vegetable samples affected the extraction of $\mathrm{Fe}$ in vegetable samples when HF was not used. Sucharová and Suchara ${ }^{10}$ have shown that $\mathrm{Si}$ affects recoveries of several elements in plant materials, e.g., $\mathrm{Cu}, \mathrm{Fe}, \mathrm{Ni}, \mathrm{Zn}$, etc., depending on its concentration.
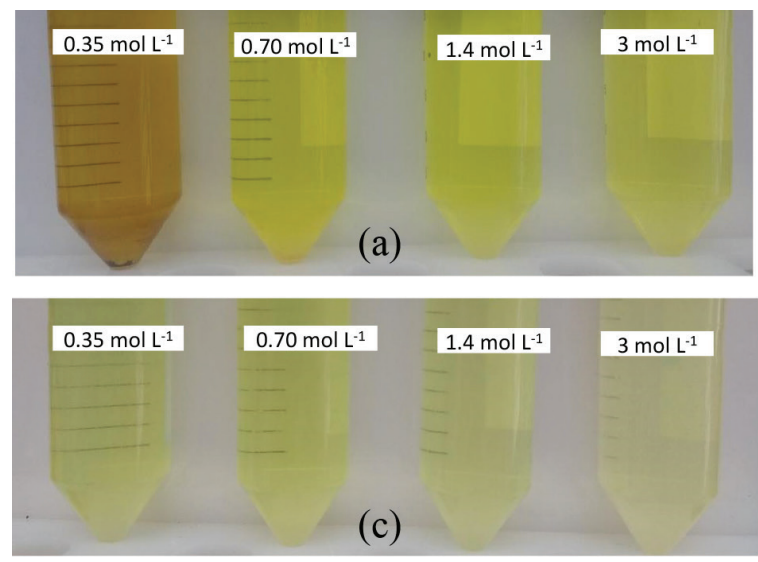

The validation and quality assurance of recoveries can be confirmed using reference materials and the range of acceptance values (80 to $110 \%$ ). According to Tavernies et al. ${ }^{11}$ certified reference materials are useful tools to achieve traceability in analytical measurements, calibrate equipment and methods, monitor laboratory performance, allowing to compare methods and their validations and quality assurance. Considering this validation factor and the recoveries obtained from 80 to $110 \%$, it can be concluded that these data are valid.

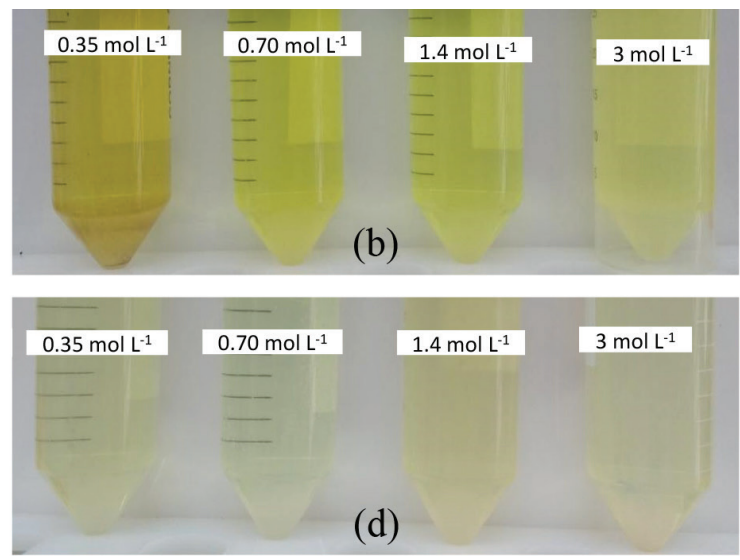

Figure 2. Digests obtained using MAXI 44 vessels and different nitric acid concentrations at $200{ }^{\circ} \mathrm{C}$. (a) Bovine liver digests without $\mathrm{H}_{2} \mathrm{O}_{2}$; (b) bovine liver digests with $\mathrm{H}_{2} \mathrm{O}_{2}$; (c) rice flour digests without $\mathrm{H}_{2} \mathrm{O}_{2}$; (d) rice flour digests with $\mathrm{H}_{2} \mathrm{O}_{2}$.

Table 4. Recoveries of some elements in digests of bovine liver samples obtained using different vessels and concentrations of $\mathrm{HNO}_{3}$ with or without $\mathrm{H}_{2} \mathrm{O}_{2}$ at 200 and $240{ }^{\circ} \mathrm{C}$

\begin{tabular}{|c|c|c|c|c|c|c|c|c|c|c|}
\hline \multirow{2}{*}{ Rotor } & \multirow{2}{*}{ Temperature $/{ }^{\circ} \mathrm{C}$} & \multirow{2}{*}{ Medium } & \multicolumn{8}{|c|}{ Recovery / \% } \\
\hline & & & $\mathrm{Ca}$ & $\mathrm{Cu}$ & $\mathrm{Fe}$ & $\mathrm{K}$ & $\mathrm{Mg}$ & $\mathrm{Na}$ & $\mathrm{P}$ & $\mathrm{Zn}$ \\
\hline MAXI 44 & \multirow{4}{*}{200} & $3.0 \mathrm{~mol} \mathrm{~L}^{-1}$ & $98 \pm 3$ & $104 \pm 2$ & $81 \pm 1$ & $99 \pm 1$ & $85 \pm 1$ & $74 \pm 0$ & $74 \pm 0$ & $85 \pm 2$ \\
\hline MAXI 44 & & $1.4 \mathrm{~mol} \mathrm{~L}^{-1}$ & $71 \pm 6$ & $105 \pm 1$ & $84 \pm 1$ & $107 \pm 1$ & $87 \pm 1$ & $78 \pm 1$ & $79 \pm 1$ & $88 \pm 3$ \\
\hline MAXI 44 & & $0.7 \mathrm{~mol} \mathrm{~L}^{-1}$ & $106 \pm 21$ & $104 \pm 0$ & $84 \pm 0$ & $109 \pm 1$ & $88 \pm 1$ & $80 \pm 0$ & $81 \pm 0$ & $93 \pm 1$ \\
\hline MAXI 44 & & $0.35 \mathrm{~mol} \mathrm{~L}^{-1}$ & $61 \pm 2$ & $60 \pm 7$ & $76 \pm 2$ & $106 \pm 1$ & $87 \pm 0$ & $79 \pm 0$ & $82 \pm 1$ & $92 \pm 5$ \\
\hline MAXI 44 & \multirow{4}{*}{200} & $3.0 \mathrm{~mol} \mathrm{~L}^{-1}+\mathrm{H}_{2} \mathrm{O}_{2}$ & $92 \pm 9$ & $104 \pm 0$ & $80 \pm 0$ & $95 \pm 0$ & $83 \pm 1$ & $76 \pm 0$ & $78 \pm 1$ & $79 \pm 1$ \\
\hline MAXI 44 & & $1.4 \mathrm{~mol} \mathrm{~L}^{-1}+\mathrm{H}_{2} \mathrm{O}_{2}$ & $82 \pm 1$ & $102 \pm 0$ & $81 \pm 0$ & $97 \pm 0$ & $85 \pm 0$ & $77 \pm 0$ & $81 \pm 1$ & $85 \pm 1$ \\
\hline MAXI 44 & & $0.7 \mathrm{~mol} \mathrm{~L}^{-1}+\mathrm{H}_{2} \mathrm{O}_{2}$ & $76 \pm 6$ & $104 \pm 3$ & $80 \pm 2$ & $103 \pm 3$ & $86 \pm 2$ & $80 \pm 2$ & $85 \pm 1$ & $89 \pm 2$ \\
\hline MAXI 44 & & $0.35 \mathrm{~mol} \mathrm{~L}^{-1}+\mathrm{H}_{2} \mathrm{O}_{2}$ & $87 \pm 1$ & $97 \pm 0$ & $78 \pm 1$ & $102 \pm 1$ & $85 \pm 0$ & $79 \pm 0$ & $84 \pm 0$ & $86 \pm 1$ \\
\hline SK-15 & \multirow{3}{*}{200} & $3.0 \mathrm{~mol} \mathrm{~L}^{-1}$ & $99 \pm 18$ & $112 \pm 3$ & $101 \pm 4$ & $84 \pm 1$ & $94 \pm 0$ & $86 \pm 1$ & $75 \pm 1$ & $105 \pm 6$ \\
\hline SK-15 & & $1.4 \mathrm{~mol} \mathrm{~L}^{-1}$ & $89 \pm 2$ & $107 \pm 1$ & $85 \pm 15$ & $84 \pm 0$ & $95 \pm 0$ & $86 \pm 0$ & $77 \pm 0$ & $86 \pm 2$ \\
\hline SK-15 & & $0.7 \mathrm{~mol} \mathrm{~L}^{-1}$ & $97 \pm 4$ & $87 \pm 5$ & $7 \pm 3$ & $87 \pm 3$ & $95 \pm 1$ & $86 \pm 2$ & $79 \pm 2$ & $110 \pm 0$ \\
\hline SK-15 & \multirow{4}{*}{200} & $3.0 \mathrm{~mol} \mathrm{~L}^{-1}+\mathrm{H}_{2} \mathrm{O}_{2}$ & $106 \pm 4$ & $113 \pm 1$ & $100 \pm 2$ & $90 \pm 2$ & $95 \pm 0$ & $90 \pm 1$ & $81 \pm 0$ & $117 \pm 1$ \\
\hline SK-15 & & $1.4 \mathrm{~mol} \mathrm{~L}^{-1}+\mathrm{H}_{2} \mathrm{O}_{2}$ & $73 \pm 5$ & $108 \pm 4$ & $100 \pm 1$ & $90 \pm 4$ & $96 \pm 1$ & $83 \pm 3$ & $81 \pm 1$ & $117 \pm 0$ \\
\hline SK-15 & & $0.7 \mathrm{~mol} \mathrm{~L}^{-1}+\mathrm{H}_{2} \mathrm{O}_{2}$ & $85 \pm 1$ & $95 \pm 3$ & $4 \pm 4$ & $81 \pm 3$ & $93 \pm 1$ & $77 \pm 2$ & $78 \pm 0$ & $117 \pm 2$ \\
\hline SK-15 & & $0.35 \mathrm{~mol} \mathrm{~L}^{-1}+\mathrm{H}_{2} \mathrm{O}_{2}$ & $80 \pm 1$ & $67 \pm 2$ & $44 \pm 7$ & $82 \pm 5$ & $92 \pm 0$ & $77 \pm 6$ & $79 \pm 2$ & $108 \pm 4$ \\
\hline SRC & \multirow{4}{*}{200} & $3.0 \mathrm{~mol} \mathrm{~L}^{-1}+\mathrm{H}_{2} \mathrm{O}_{2}$ & $85 \pm 5$ & $102 \pm 0$ & $83 \pm 0$ & $92 \pm 0$ & $85 \pm 1$ & $77 \pm 0$ & $84 \pm 1$ & $90 \pm 1$ \\
\hline SRC & & $1.4 \mathrm{~mol} \mathrm{~L}^{-1}+\mathrm{H}_{2} \mathrm{O}_{2}$ & $98 \pm 3$ & $101 \pm 2$ & $4 \pm 0$ & $95 \pm 2$ & $88 \pm 1$ & $79 \pm 1$ & $92 \pm 1$ & $97 \pm 1$ \\
\hline SRC & & $0.7 \mathrm{~mol} \mathrm{~L}^{-1}+\mathrm{H}_{2} \mathrm{O}_{2}$ & $75 \pm 8$ & $81 \pm 5$ & $8 \pm 3$ & $98 \pm 2$ & $89 \pm 1$ & $80 \pm 2$ & $94 \pm 2$ & $96 \pm 1$ \\
\hline SRC & & $0.35 \mathrm{~mol} \mathrm{~L}^{-1}+\mathrm{H}_{2} \mathrm{O}_{2}$ & $74 \pm 4$ & $36 \pm 3$ & $26 \pm 3$ & $101 \pm 1$ & $90 \pm 1$ & $80 \pm 1$ & $95 \pm 1$ & $83 \pm 1$ \\
\hline SRC & \multirow{3}{*}{240} & $3.0 \mathrm{~mol} \mathrm{~L}^{-1}+\mathrm{H}_{2} \mathrm{O}_{2}$ & $87 \pm 1$ & $87 \pm 1$ & $90 \pm 0$ & $101 \pm 1$ & $92 \pm 2$ & $80 \pm 1$ & $95 \pm 1$ & $87 \pm 1$ \\
\hline SRC & & $1.4 \mathrm{~mol} \mathrm{~L}^{-1}+\mathrm{H}_{2} \mathrm{O}_{2}$ & $100 \pm 2$ & $95 \pm 0$ & $3 \pm 2$ & $81 \pm 2$ & $98 \pm 0$ & $83 \pm 0$ & $76 \pm 1$ & $98 \pm 0$ \\
\hline SRC & & $0.7 \mathrm{~mol} \mathrm{~L}^{-1}+\mathrm{H}_{2} \mathrm{O}_{2}$ & $80 \pm 2$ & $65 \pm 8$ & ND & $91 \pm 2$ & $96 \pm 4$ & $80 \pm 3$ & $76 \pm 2$ & $96 \pm 3$ \\
\hline
\end{tabular}

SRC: single reaction chamber; ND: not determined. 
Table 5. Recoveries of some elements in digests of rice flour samples obtained using different vessels and concentrations of $\mathrm{HNO}_{3}$ with or without $\mathrm{H}_{2} \mathrm{O}_{2}$ at 200 and $240{ }^{\circ} \mathrm{C}$

\begin{tabular}{|c|c|c|c|c|c|c|c|c|c|c|c|}
\hline \multirow{2}{*}{ Rotor } & \multirow{2}{*}{ Temperature $/{ }^{\circ} \mathrm{C}$} & \multirow{2}{*}{ Medium } & \multicolumn{9}{|c|}{ Recovery / \% } \\
\hline & & & $\mathrm{Ca}$ & $\mathrm{Cu}$ & $\mathrm{Fe}$ & $\mathrm{K}$ & $\mathrm{Mg}$ & $\mathrm{Mn}$ & $\mathrm{Na}$ & $\mathrm{P}$ & $\mathrm{Zn}$ \\
\hline MAXI 44 & \multirow{4}{*}{200} & $3.0 \mathrm{~mol} \mathrm{~L}^{-1}$ & $94 \pm 1$ & $93 \pm 17$ & $65 \pm 4$ & $80 \pm 1$ & $98 \pm 2$ & $110 \pm 2$ & $88 \pm 0$ & $90 \pm 1$ & $96 \pm 4$ \\
\hline MAXI 44 & & $1.4 \mathrm{~mol} \mathrm{~L}^{-1}$ & $86 \pm 0$ & $74 \pm 2$ & $80 \pm 19$ & $83 \pm 3$ & $91 \pm 7$ & $108 \pm 1$ & $87 \pm 0$ & $94 \pm 0$ & $84 \pm 0$ \\
\hline MAXI 44 & & $0.7 \mathrm{~mol} \mathrm{~L}^{-1}$ & $74 \pm 8$ & $88 \pm 2$ & $81 \pm 26$ & $87 \pm 1$ & $92 \pm 2$ & $109 \pm 1$ & $88 \pm 1$ & $97 \pm 1$ & $93 \pm 6$ \\
\hline MAXI 44 & & $0.35 \mathrm{~mol} \mathrm{~L}^{-1}$ & $91 \pm 5$ & $55 \pm 37$ & $42 \pm 8$ & $84 \pm 2$ & $102 \pm 1$ & $109 \pm 2$ & $93 \pm 45$ & $97 \pm 0$ & $99 \pm 6$ \\
\hline MAXI 44 & \multirow{4}{*}{200} & $3.0 \mathrm{~mol} \mathrm{~L}^{-1}+\mathrm{H}_{2} \mathrm{O}_{2}$ & $107 \pm 4$ & $84 \pm 1$ & $73 \pm 15$ & $84 \pm 3$ & $86 \pm 5$ & $108 \pm 1$ & $108 \pm 1$ & $101 \pm 1$ & $96 \pm 3$ \\
\hline MAXI 44 & & $1.4 \mathrm{~mol} \mathrm{~L}^{-1}+\mathrm{H}_{2} \mathrm{O}_{2}$ & $103 \pm 4$ & $92 \pm 3$ & $67 \pm 2$ & $83 \pm 3$ & $96 \pm 4$ & $109 \pm 4$ & $95 \pm 2$ & $103 \pm 3$ & $104 \pm 7$ \\
\hline MAXI 44 & & $0.7 \mathrm{~mol} \mathrm{~L}^{-1}+\mathrm{H}_{2} \mathrm{O}_{2}$ & $102 \pm 2$ & $91 \pm 2$ & $67 \pm 3$ & $89 \pm 2$ & $95 \pm 3$ & $110 \pm 4$ & $105 \pm 7$ & $106 \pm 4$ & $95 \pm 2$ \\
\hline MAXI 44 & & $0.35 \mathrm{~mol} \mathrm{~L}^{-1}+\mathrm{H}_{2} \mathrm{O}_{2}$ & $99 \pm 1$ & $72 \pm 0$ & $48 \pm 3$ & $89 \pm 2$ & $92 \pm 2$ & $108 \pm 3$ & $102 \pm 6$ & $109 \pm 3$ & $93 \pm 2$ \\
\hline SK-15 & \multirow{4}{*}{200} & $3.0 \mathrm{~mol} \mathrm{~L}^{-1}$ & $98 \pm 2$ & $83 \pm 3$ & $80 \pm 43$ & $88 \pm 4$ & $94 \pm 2$ & $113 \pm 1$ & $84 \pm 5$ & $95 \pm 3$ & $102 \pm 8$ \\
\hline SK-15 & & $1.4 \mathrm{~mol} \mathrm{~L}^{-1}$ & $112 \pm 17$ & $83 \pm 3$ & $78 \pm 13$ & $86 \pm 2$ & $96 \pm 4$ & $112 \pm 1$ & $85 \pm 7$ & $94 \pm 0$ & $95 \pm 0$ \\
\hline SK-15 & & $0.7 \mathrm{~mol} \mathrm{~L}^{-1}$ & $109 \pm 13$ & $78 \pm 3$ & $65 \pm 7$ & $90 \pm 2$ & $97 \pm 3$ & $115 \pm 2$ & $84 \pm 2$ & $97 \pm 0$ & $103 \pm 4$ \\
\hline SK-15 & & $0.35 \mathrm{~mol} \mathrm{~L}^{-1}$ & $110 \pm 11$ & $16 \pm 4$ & ND & $87 \pm 2$ & $97 \pm 4$ & $112 \pm 2$ & $93 \pm 1$ & $96 \pm 2$ & $84 \pm 3$ \\
\hline SK-15 & \multirow{4}{*}{200} & $3.0 \mathrm{~mol} \mathrm{~L}^{-1}+\mathrm{H}_{2} \mathrm{O}_{2}$ & $106 \pm 19$ & $86 \pm 1$ & $63 \pm 1$ & $86 \pm 1$ & $98 \pm 2$ & $112 \pm 1$ & $106 \pm 4$ & $96 \pm 1$ & $87 \pm 6$ \\
\hline SK-15 & & $1.4 \mathrm{~mol} \mathrm{~L}^{-1}+\mathrm{H}_{2} \mathrm{O}_{2}$ & $109 \pm 5$ & $81 \pm 0$ & $63 \pm 1$ & $87 \pm 1$ & $91 \pm 1$ & $107 \pm 1$ & $106 \pm 2$ & $96 \pm 1$ & $106 \pm 3$ \\
\hline SK-15 & & $0.7 \mathrm{~mol} \mathrm{~L}^{-1}+\mathrm{H}_{2} \mathrm{O}_{2}$ & $109 \pm 3$ & $85 \pm 1$ & $61 \pm 3$ & $91 \pm 2$ & $98 \pm 0$ & $113 \pm 1$ & $103 \pm 0$ & $100 \pm 1$ & $101 \pm 1$ \\
\hline$\underline{\text { SK-15 }}$ & & $0.35 \mathrm{~mol} \mathrm{~L}^{-1}+\mathrm{H}_{2} \mathrm{O}_{2}$ & $105 \pm 11$ & $44 \pm 1$ & $42 \pm 2$ & $90 \pm 1$ & $96 \pm 0$ & $112 \pm 1$ & $99 \pm 0$ & $101 \pm 1$ & $72 \pm 0$ \\
\hline SRC & \multirow{4}{*}{200} & $3.0 \mathrm{~mol} \mathrm{~L}^{-1}+\mathrm{H}_{2} \mathrm{O}_{2}$ & $76 \pm 2$ & $63 \pm 0$ & $52 \pm 1$ & $70 \pm 0$ & $75 \pm 1$ & $89 \pm 0$ & $61 \pm 1$ & $80 \pm 1$ & $79 \pm 2$ \\
\hline SRC & & $1.4 \mathrm{~mol} \mathrm{~L}^{-1}+\mathrm{H}_{2} \mathrm{O}_{2}$ & $73 \pm 1$ & $68 \pm 2$ & $50 \pm 1$ & $72 \pm 3$ & $82 \pm 2$ & $90 \pm 3$ & $55 \pm 3$ & $81 \pm 2$ & $79 \pm 0$ \\
\hline SRC & & $0.7 \mathrm{~mol} \mathrm{~L}^{-1}+\mathrm{H}_{2} \mathrm{O}_{2}$ & $75 \pm 5$ & $38 \pm 10$ & ND & $76 \pm 4$ & $80 \pm 2$ & $93 \pm 5$ & $65 \pm 8$ & $88 \pm 6$ & $81 \pm 3$ \\
\hline SRC & & $0.35 \mathrm{~mol} \mathrm{~L}^{-1}+\mathrm{H}_{2} \mathrm{O}_{2}$ & $87 \pm 15$ & $5 \pm 7$ & ND & $82 \pm 8$ & $84 \pm 8$ & $98 \pm 7$ & $85 \pm 21$ & $94 \pm 7$ & $88 \pm 5$ \\
\hline SRC & \multirow{3}{*}{240} & $3.0 \mathrm{~mol} \mathrm{~L}^{-1}+\mathrm{H}_{2} \mathrm{O}_{2}$ & $110 \pm 10$ & $107 \pm 9$ & $84 \pm 5$ & $82 \pm 4$ & $95 \pm 5$ & $75 \pm 3$ & $94 \pm 18$ & $124 \pm 10$ & $117 \pm 9$ \\
\hline SRC & & $1.4 \mathrm{~mol} \mathrm{~L}^{-1}+\mathrm{H}_{2} \mathrm{O}_{2}$ & $115 \pm 27$ & $91 \pm 3$ & $63 \pm 4$ & $83 \pm 3$ & $85 \pm 1$ & $116 \pm 3$ & $67 \pm 7$ & $109 \pm 4$ & $113 \pm 14$ \\
\hline SRC & & $0.7 \mathrm{~mol} \mathrm{~L}^{-1}+\mathrm{H}_{2} \mathrm{O}_{2}$ & $106 \pm 2$ & $56 \pm 2$ & $13 \pm 1$ & $84 \pm 4$ & $85 \pm 1$ & $116 \pm 2$ & $65 \pm 3$ & $112 \pm 2$ & $110 \pm 2$ \\
\hline
\end{tabular}

SRC: single reaction chamber; ND: not determined.

Residual carbon contents were in the range of 33 to $96 \%$ for all bovine liver and rice flour digests for all tested concentrations of $\mathrm{HNO}_{3}$, heating program and $\mathrm{H}_{2} \mathrm{O}_{2}$ addition (Table 6). However, these values were not determined when digests contained appreciable amounts of solid residues, clearly indicating an inefficient digestion as observed when using $0.35 \mathrm{~mol} \mathrm{~L}^{-1} \mathrm{HNO}_{3}$. For both samples, RCC values were around 33 to $52 \%$ when using $\mathrm{H}_{2} \mathrm{O}_{2}$. RCC varied from 53 to $96 \%$ without using $\mathrm{H}_{2} \mathrm{O}_{2}$. This large difference is related to the action of $\mathrm{H}_{2} \mathrm{O}_{2}$ as auxiliary reagent, incrementing the nitric acid regeneration and, consequently, facilitating the digestion process. ${ }^{12}$

As shown in Table 6, residual acidities using the MAXI 44 rotor for rice flour digestions with $\mathrm{H}_{2} \mathrm{O}_{2}$ were around $100 \%$ and for bovine liver were around $90 \%$. These values were around 100 and $80 \%$, respectively, without using $\mathrm{H}_{2} \mathrm{O}_{2}$. Both conditions indicated that, as expected, nitric acid was regenerated during the digestion process and this effect is related to the vessel shape and gradients of temperature in the gas phase, liquid phase and tube walls, particularly during the initial step of microwave-assisted heating process. Bizzi et al..$^{13}$ have clearly demonstrated these effects by measuring gas and liquid phases temperatures along the heating program using thermographic images and a sapphire thermocouple.

\section{Analytical performance: SK-15}

The digests of bovine liver samples obtained using the SK-15 rotor without $\mathrm{H}_{2} \mathrm{O}_{2}$ (Figure 3a) presented dark solid particles when using $0.7 \mathrm{~mol} \mathrm{~L}^{-1} \mathrm{HNO}_{3}$. Based on this observation, digestion was not evaluated using $0.35 \mathrm{~mol} \mathrm{~L}^{-1}$ nitric acid solution. The presence of solid residues after digestion can be explained because bovine liver contains higher variety of lipid structures than rice flour. ${ }^{14}$ However, digests had a better visual aspect when using the mixture composed by nitric acid plus hydrogen peroxide (Figure $3 b$ ). It can be seen in Table 4 that best recoveries were reached when using 3 and $1.4 \mathrm{~mol} \mathrm{~L}^{-1} \mathrm{HNO}_{3}$ plus $\mathrm{H}_{2} \mathrm{O}_{2}$.

On the other hand, the digests of rice flour samples obtained without $\mathrm{H}_{2} \mathrm{O}_{2}$ in the same conditions of heating program and nitric acid concentrations had a good visual aspect, except for $0.35 \mathrm{~mol} \mathrm{~L}^{-1} \mathrm{HNO}_{3}$ (Figure 3c). As previously observed for bovine liver, visual aspect of rice flour digests was improved when hydrogen peroxide was 
Table 6. Residual acidity and residual carbon content (RCC) of digests of rice flour and bovine liver samples obtained using different vessels and temperatures of 200 and $240{ }^{\circ} \mathrm{C}$

\begin{tabular}{|c|c|c|c|c|c|c|}
\hline \multirow{2}{*}{ Rotor } & \multirow{2}{*}{ Temperature $/{ }^{\circ} \mathrm{C}$} & \multirow{2}{*}{ Medium } & \multicolumn{2}{|c|}{ Residual acidity / \% } & \multicolumn{2}{|c|}{$\mathrm{RCC} / \%$} \\
\hline & & & Rice flour & Bovine liver & Rice flour & Bovine liver \\
\hline MAXI 44 & \multirow{4}{*}{200} & $3.0 \mathrm{~mol} \mathrm{~L}^{-1}$ & 95 & 92 & $86 \pm 4$ & $53 \pm 5$ \\
\hline MAXI 44 & & $1.4 \mathrm{~mol} \mathrm{~L}^{-1}$ & 107 & 75 & $96 \pm 1$ & $67 \pm 5$ \\
\hline MAXI 44 & & $0.7 \mathrm{~mol} \mathrm{~L}^{-1}$ & 100 & 71 & $93 \pm 4$ & $67 \pm 1$ \\
\hline MAXI 44 & & $0.35 \mathrm{~mol} \mathrm{~L}^{-1}$ & 57 & 71 & $84 \pm 1$ & b \\
\hline MAXI 44 & \multirow{4}{*}{200} & $3.0 \mathrm{~mol} \mathrm{~L}^{-1}+\mathrm{H}_{2} \mathrm{O}_{2}$ & 92 & 100 & $33 \pm 1$ & $42 \pm 1$ \\
\hline MAXI 44 & & $1.4 \mathrm{~mol} \mathrm{~L}^{-1}+\mathrm{H}_{2} \mathrm{O}_{2}$ & 123 & 82 & $52 \pm 26$ & $45 \pm 1$ \\
\hline MAXI 44 & & $0.7 \mathrm{~mol} \mathrm{~L}^{-1}+\mathrm{H}_{2} \mathrm{O}_{2}$ & 82 & 82 & $40 \pm 3$ & $50 \pm 1$ \\
\hline MAXI 44 & & $0.35 \mathrm{~mol} \mathrm{~L}^{-1}+\mathrm{H}_{2} \mathrm{O}_{2}$ & 107 & 107 & $45 \pm 1$ & b \\
\hline SK-15 & \multirow{4}{*}{200} & $3.0 \mathrm{~mol} \mathrm{~L}^{-1}$ & 55 & 73 & $44 \pm 14$ & $41 \pm 1$ \\
\hline SK-15 & & $1.4 \mathrm{~mol} \mathrm{~L}^{-1}$ & 82 & 43 & $72 \pm 5$ & $55 \pm 5$ \\
\hline SK-15 & & $0.7 \mathrm{~mol} \mathrm{~L}^{-1}$ & 57 & 43 & $79 \pm 6$ & b \\
\hline SK-15 & & $0.35 \mathrm{~mol} \mathrm{~L}^{-1}$ & 57 & a & b & a \\
\hline SK-15 & \multirow{4}{*}{200} & $3.0 \mathrm{~mol} \mathrm{~L}^{-1}+\mathrm{H}_{2} \mathrm{O}_{2}$ & 83 & 94 & $25 \pm 1$ & $33 \pm 1$ \\
\hline SK-15 & & $1.4 \mathrm{~mol} \mathrm{~L}^{-1}+\mathrm{H}_{2} \mathrm{O}_{2}$ & 91 & 82 & $32 \pm 1$ & $37 \pm 1$ \\
\hline SK-15 & & $0.7 \mathrm{~mol} \mathrm{~L}^{-1}+\mathrm{H}_{2} \mathrm{O}_{2}$ & 55 & 55 & $31 \pm 1$ & $40 \pm 0$ \\
\hline$\underline{\text { SK-15 }}$ & & $0.35 \mathrm{~mol} \mathrm{~L}^{-1}+\mathrm{H}_{2} \mathrm{O}_{2}$ & 107 & 143 & b & b \\
\hline SRC & \multirow{4}{*}{200} & $3.0 \mathrm{~mol} \mathrm{~L}^{-1}+\mathrm{H}_{2} \mathrm{O}_{2}$ & 50 & 65 & $7 \pm 0$ & $22 \pm 0$ \\
\hline $\mathrm{SRC}$ & & $1.4 \mathrm{~mol} \mathrm{~L}^{-1}+\mathrm{H}_{2} \mathrm{O}_{2}$ & 27 & 36 & $12 \pm 1$ & $35 \pm 0$ \\
\hline SRC & & $0.7 \mathrm{~mol} \mathrm{~L}^{-1}+\mathrm{H}_{2} \mathrm{O}_{2}$ & 27 & 55 & b & b \\
\hline SRC & & $0.35 \mathrm{~mol} \mathrm{~L}^{-1}+\mathrm{H}_{2} \mathrm{O}_{2}$ & 54 & 71 & b & $\mathrm{b}$ \\
\hline SRC & \multirow{3}{*}{240} & $3.0 \mathrm{~mol} \mathrm{~L}^{-1}+\mathrm{H}_{2} \mathrm{O}_{2}$ & 38 & 52 & $1 \pm 0$ & $9 \pm 0$ \\
\hline $\mathrm{SRC}$ & & $1.4 \mathrm{~mol} \mathrm{~L}^{-1}+\mathrm{H}_{2} \mathrm{O}_{2}$ & 32 & 41 & $4 \pm 0$ & $23 \pm 4$ \\
\hline SRC & & $0.7 \mathrm{~mol} \mathrm{~L}^{-1}+\mathrm{H}_{2} \mathrm{O}_{2}$ & 54 & 45 & $8 \pm 1$ & $56 \pm 2$ \\
\hline
\end{tabular}

${ }^{a}$ Determinations were not made in these conditions; ${ }^{b}$ solid dark particles in digests. SRC: single reaction chamber.
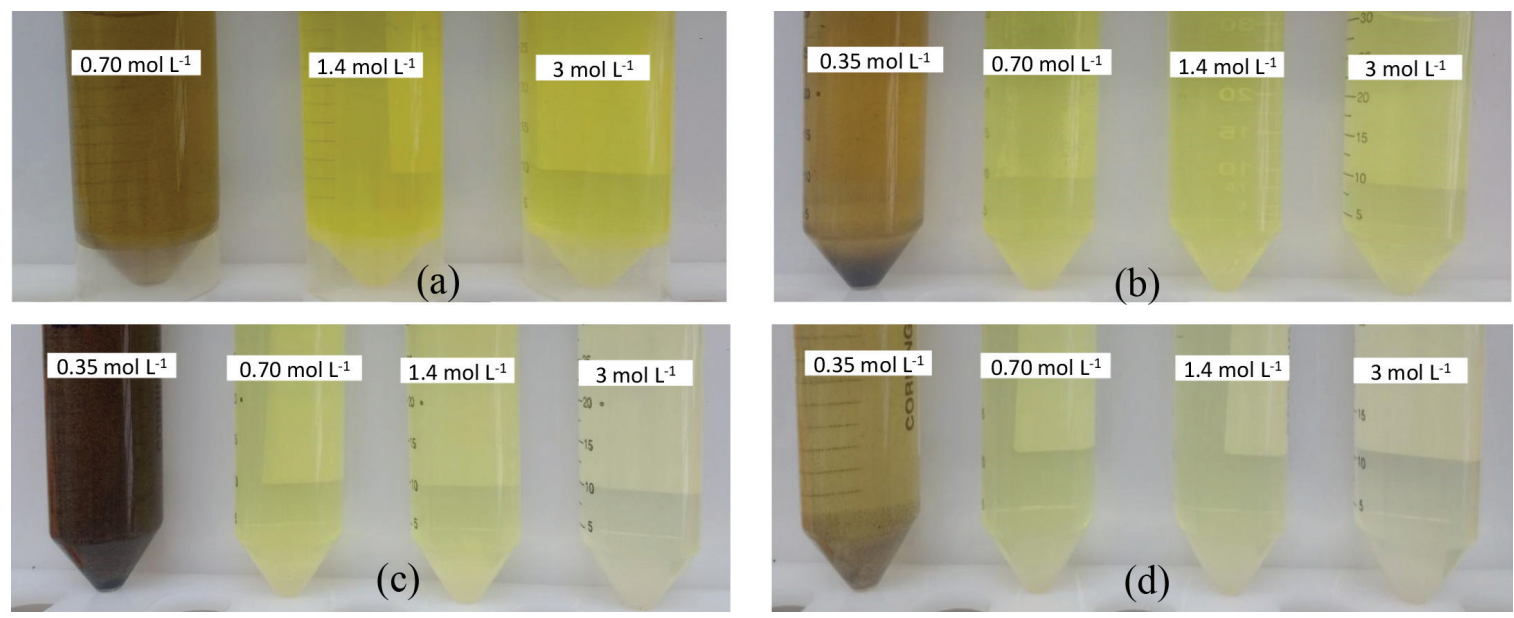

Figure 3. Digests obtained using SK-15 vessels and different nitric acid concentrations at $200{ }^{\circ} \mathrm{C}$. (a) Bovine liver digests without $\mathrm{H}_{2} \mathrm{O}_{2}$; (b) bovine liver digests with $\mathrm{H}_{2} \mathrm{O}_{2}$; (c) rice flour digests without $\mathrm{H}_{2} \mathrm{O}_{2}$; (d) rice flour digests with $\mathrm{H}_{2} \mathrm{O}_{2}$.

added to nitric acid solution for promoting digestions. In general, both digestion conditions led to good recoveries for all tested nitric acid solutions (Table 5), however, recoveries of $\mathrm{Fe}$ and $\mathrm{Cu}$ in $0.35 \mathrm{~mol} \mathrm{~L}^{-1} \mathrm{HNO}_{3}$ were not quantitative. It is known that $\mathrm{Fe}$ is not easily recovered from plant tissues due to combination with matrix elements, particularly $\mathrm{Si}^{10,11}$
The residual acidities for digestions with $\mathrm{HNO}_{3}$ plus $\mathrm{H}_{2} \mathrm{O}_{2}$ of rice flour and bovine liver were in the range of 82 to $143 \%$. These values decreased up to $43 \%$ when digestion was performed without $\mathrm{H}_{2} \mathrm{O}_{2}$. This behavior is expected considering the mechanism involved for regeneration of nitric acid and its dependence on the amount of oxygen available in the closed vessel. ${ }^{12}$ 
The RCC was analyzed only for digestions without solid residues in the final solution. RCC were around $40 \%$ for rice flour and bovine liver digests with $3 \mathrm{~mol} \mathrm{~L}^{-1} \mathrm{HNO}_{3}$ without using $\mathrm{H}_{2} \mathrm{O}_{2}$ (Table 6). For digestions performed using lower concentrations of $\mathrm{HNO}_{3}\left(1.4\right.$ and $\left.0.7 \mathrm{~mol} \mathrm{~L}^{-1}\right)$ without $\mathrm{H}_{2} \mathrm{O}_{2}$, RCCs of rice flour samples were around 71 and $78 \%$ and for bovine liver 55\%. It can be observed that the RCC of digests obtained using the SK-15 vessels for rice flour and bovine liver samples with 3, 1.4 and $0.7 \mathrm{~mol} \mathrm{~L}^{-1} \mathrm{HNO}_{3}$ plus $\mathrm{H}_{2} \mathrm{O}_{2}$ resulted in RCCs below $40 \%$.

\section{Analytical performance: SRC technology}

According to Figure 4a, bovine liver digests obtained using the $\mathrm{SRC}$ at $200{ }^{\circ} \mathrm{C}$ and 0.35 and $0.7 \mathrm{~mol} \mathrm{~L}^{-1} \mathrm{HNO}_{3}$ plus $\mathrm{H}_{2} \mathrm{O}_{2}$ had appreciable amounts of solid residues. At higher concentrations of nitric acid, digests had better visual aspect with lower amount of solid residues. As it can be observed in Table 4, in general, quantitative recoveries were obtained for all nitric acid solutions, except for $\mathrm{Ca}$ and $\mathrm{Fe}$ for most nitric acid solutions and $\mathrm{Cu}$ in $0.35 \mathrm{~mol} \mathrm{~L}^{-1}$ nitric acid.

As it can be seen in Figure $4 \mathrm{~b}$, rice flour digests obtained at $200{ }^{\circ} \mathrm{C}$ using nitric acid plus $\mathrm{H}_{2} \mathrm{O}_{2}$ had better visual aspect in 3 and $1.4 \mathrm{~mol} \mathrm{~L}^{-1}$ of $\mathrm{HNO}_{3}$ solutions, however, 0.70 and $0.35 \mathrm{~mol} \mathrm{~L}^{-1}$ acid solutions contained residual solids. As shown in Table 5, in general, quantitative recoveries were reached for most elements, except for $\mathrm{Fe}, \mathrm{Na}$ and $\mathrm{Cu}$, in all nitric acid solutions.

Figures $4 \mathrm{c}$ and $4 \mathrm{~d}$ show better visual aspect for rice flour and bovine liver digests obtained applying a maximum temperature of $240{ }^{\circ} \mathrm{C}$ compared to digests obtained at $200{ }^{\circ} \mathrm{C}$, but solid residues still remained when using $0.70 \mathrm{~mol} \mathrm{~L}^{-1} \mathrm{HNO}_{3}$. At $240{ }^{\circ} \mathrm{C}, 0.35 \mathrm{~mol} \mathrm{~L}^{-1} \mathrm{HNO}_{3}$ was not tested because even when using $0.70 \mathrm{~mol} \mathrm{~L}^{-1} \mathrm{HNO}_{3}$ digestion was not efficient. As observed in Table 5 for rice flour digests, using $3 \mathrm{~mol} \mathrm{~L}^{-1} \mathrm{HNO}_{3}$, the determination of the analytes was efficient, except for $\mathrm{Mn}$ and $\mathrm{P}$. The determination of the analytes was efficient for bovine liver digests obtained at $240{ }^{\circ} \mathrm{C}$ using 3 and $1.4 \mathrm{~mol} \mathrm{~L}^{-1} \mathrm{HNO}_{3}$, except for Fe at $1.4 \mathrm{~mol} \mathrm{~L}^{-1}$.

Analyzing all digests for both samples and using both heating programs, when applying SRC technology the acid digestion mixture should contain at least 1.4 or $3 \mathrm{~mol} \mathrm{~L}^{-1} \mathrm{HNO}_{3}$ plus $\mathrm{H}_{2} \mathrm{O}_{2}$. It was also observed that at higher temperature, i.e., $240{ }^{\circ} \mathrm{C}$, the determination of analytes was more accurate than at lower temperature, i.e., $200^{\circ} \mathrm{C}$. When the temperature increases, the oxidative power of the dilute nitric acid solution also increases. Generally, better recoveries of analytes were obtained when using 3 or $1.4 \mathrm{~mol} \mathrm{~L}^{-1} \mathrm{HNO}_{3}$ at digestion temperature of $240{ }^{\circ} \mathrm{C}$.

Residual acidities in digests obtained using the SRC technology at $200{ }^{\circ} \mathrm{C}$ were around 40 and $55 \%$ for rice flour and bovine liver, respectively. For other rice flour digestions at $240{ }^{\circ} \mathrm{C}$, residual acidities were also around $40 \%$ (Table 6). These values indicate that nitric acid regeneration was less significant than that observed when using microwave cavity conventional oven. This effect is expected taking into account the different forms of heating. It is important to remember that for the SRC technology reactions vessels are immersed in a water/acid base load that absorbs microwave radiation and transfer the heat to the solutions contained in the reaction vessels.

The percentage of RCCs was analyzed only for digests without solid residues. Rice flour samples digested at $200{ }^{\circ} \mathrm{C}$ with 3 and $1.4 \mathrm{~mol} \mathrm{~L}^{-1} \mathrm{HNO}_{3}$ presented RCCs around 7 and $12 \%$, respectively (Table 6). The RCCs for bovine liver samples digested at these same conditions
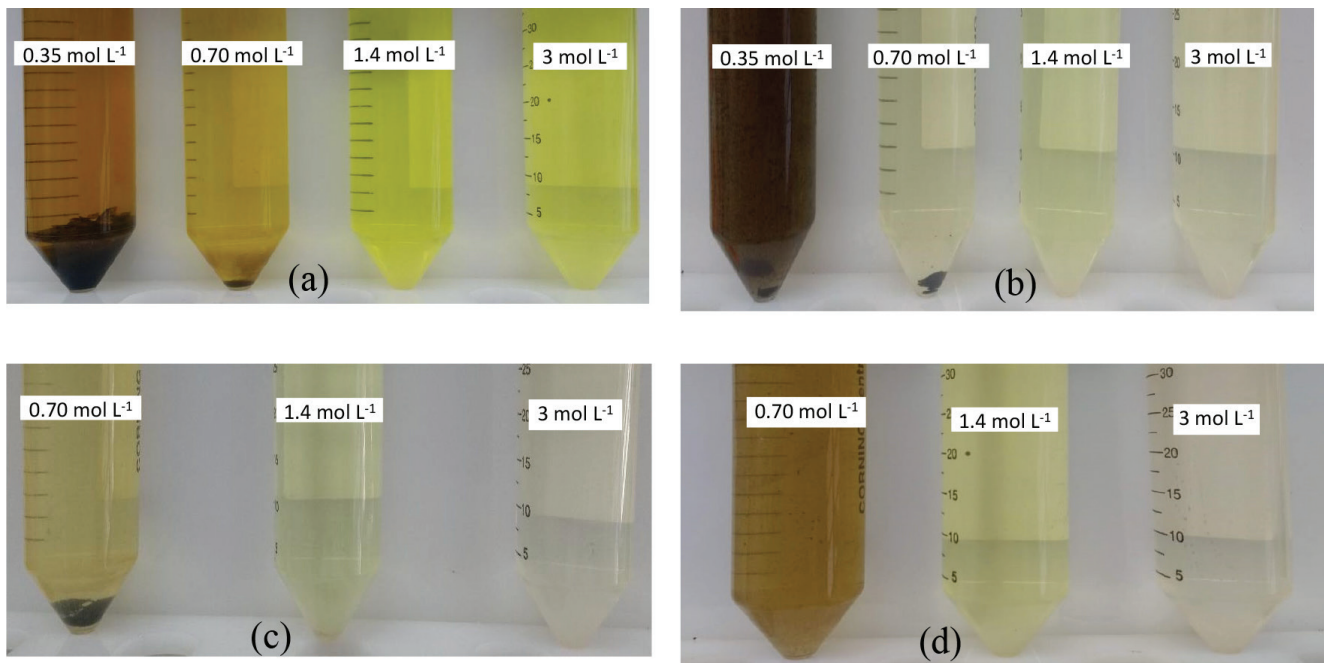

Figure 4. Digests obtained using the SRC technology and different nitric acid concentrations. (a) Bovine liver digests with $\mathrm{H}_{2} \mathrm{O}_{2}$ at $200{ }^{\circ} \mathrm{C}$; (b) rice flour digests with $\mathrm{H}_{2} \mathrm{O}_{2}$ at $200{ }^{\circ} \mathrm{C}$; (c) rice flour digests with $\mathrm{H}_{2} \mathrm{O}_{2}$ at $240{ }^{\circ} \mathrm{C}$; (d) bovine liver digests with $\mathrm{H}_{2} \mathrm{O}_{2}$ at $240{ }^{\circ} \mathrm{C}$. 
were around 22 and 35\%, respectively. By increasing the temperature to $240{ }^{\circ} \mathrm{C}$, digestions were more efficient with RCCs varying from 1 to $4 \%$ for rice flour samples and from 9 to $23 \%$ for bovine liver samples. The SRC technology led to the best RCC results when comparing all vessels evaluated.

\section{General comments}

The use of digestions vessels with different shapes and heights as shown in Figure 1, influences $\mathrm{HNO}_{3}$ condensation because taller vessels, e.g., MAXI 44, present a more pronounced gradient of temperature. As a consequence, this aspect affects the quality and efficiency of the digestion. In the SRC technology, the used vessel has $1.3 \mathrm{~cm}$ of internal diameter and $13 \mathrm{~cm}$ of height. Consequently, condensation of nitric acid is limited because of the small volume and small height of the vessel. Moreover, as previously mentioned, the heating process is different because microwave radiation is absorbed by the base load solution inserted into the SRC. On the other hand, the MAXI 44 vessels are taller with $23.6 \mathrm{~cm}$ of height and $2.3 \mathrm{~cm}$ of internal diameter. These volume and height increase the temperature gradient and improve the regeneration of nitric acid leading to a more efficient digestion as can be visually observed (Figure 2). The SK-15 vessels have about the same volume of MAXI 44, but with different dimensions: i.e., $13 \mathrm{~cm}$ of height and $3.2 \mathrm{~cm}$ of diameter. These vessels are shorter and larger, increasing the contact area of solution and decreasing the gradient of temperature. Consequently, regeneration of nitric acid is less efficient.

It can be seen in Table 6 that digests obtained using $\mathrm{H}_{2} \mathrm{O}_{2}$, in general, had lower RCCs and better acid regeneration when compared to digestions performed without using this reagent.

\section{Characterization of reactions products by ${ }^{1} \mathrm{H}$ NMR}

In order to identify the characteristics of residual organic compounds of bovine liver sample when digested with $1.4 \mathrm{~mol} \mathrm{~L}^{-1} \mathrm{HNO}_{3}$ with or without $\mathrm{H}_{2} \mathrm{O}_{2}$, the ${ }^{1} \mathrm{H}$ NMR spectra were evaluated and the presence of organic residues in aliphatic (0.7-4.5 ppm) (Figure 5a) and aromatic regions (6.0-10.2 ppm) (Figure $5 \mathrm{~b}$ ) were demonstrated. In general, reaction conditions in MAXI 44 vessel led to higher amounts of organic residues after the sample digestion compared to reaction conditions when using SK-15 and the SRC technology, which presented intermediate and smaller quantities of residues, respectively. It can be observed in Figure 5 that digestion conditions without using $\mathrm{H}_{2} \mathrm{O}_{2}$ in MAXI 44 presented more intense signals (black spectra) when compared to the spectrum of MAXI 44 in the presence of $\mathrm{H}_{2} \mathrm{O}_{2}$ (red spectra). On the other hand, both conditions, i.e., with or without $\mathrm{H}_{2} \mathrm{O}_{2}$, using the SK-15 rotor led to similar spectra. However, spectra obtained using the SRC technology were different. In Figure 5, according to the orange and yellow spectra, respectively, sample digestion using the SRC technology at $240{ }^{\circ} \mathrm{C}$ presented the lowest variety of signals when compared to the digestion using the same equipment but at $200{ }^{\circ} \mathrm{C}$ and also when compared to the others spectra. These results are expected taking into account the conditions of digestion either in each reaction vessel or in each temperature for the SRC technology. It is also important to mention that, as expected, digestion processes are dependent on closed vessels design, reagents and heating programs, but as earlier demonstrated by Würfels et al. ${ }^{15,16}$ the characteristics of the chemical compounds also exerted a critical influence on digestion efficiencies and residual compounds present in digests.

\section{Conclusions}

These simple experiments demonstrate how the digestion efficiency of samples is critically dependent on the choice of the reaction vessel. Despite using the same heating program, the digestion efficiency is dependent on the vessel design and this aspect is related to the availability of oxygen and magnitude of the gradient of temperature to allow effective regeneration of nitric acid. The occurrence of this process was more effective when working with taller vessels and when adding hydrogen peroxide to nitric acid solution. In this sense, the use of MAXI 44 rotor is beneficial when not adopting extreme conditions of temperature for promoting digestions. It was demonstrated that quantitative recoveries were obtained when using both rotors, i.e., MAXI 44 and SK-15, even when working with nitric acid concentration as low as $0.35 \mathrm{~mol} \mathrm{~L}^{-1}$ for some elements. On the other hand, when working with the SRC technology the conditions of digestions are easily improved by increasing the maximum temperature applied for performing digestions as shown in Figure 4.

The RCCs reached lower values when working with the SRC technology. This better efficiency compared to other procedures can also be checked by visual observation of the digests. The ${ }^{1} \mathrm{H}$ NMR spectra also confirm these observations.

It can be concluded that proper application of tailored procedures to specific aims is critically dependent on thoughtful evaluation of sample characteristics and equipment performance. 


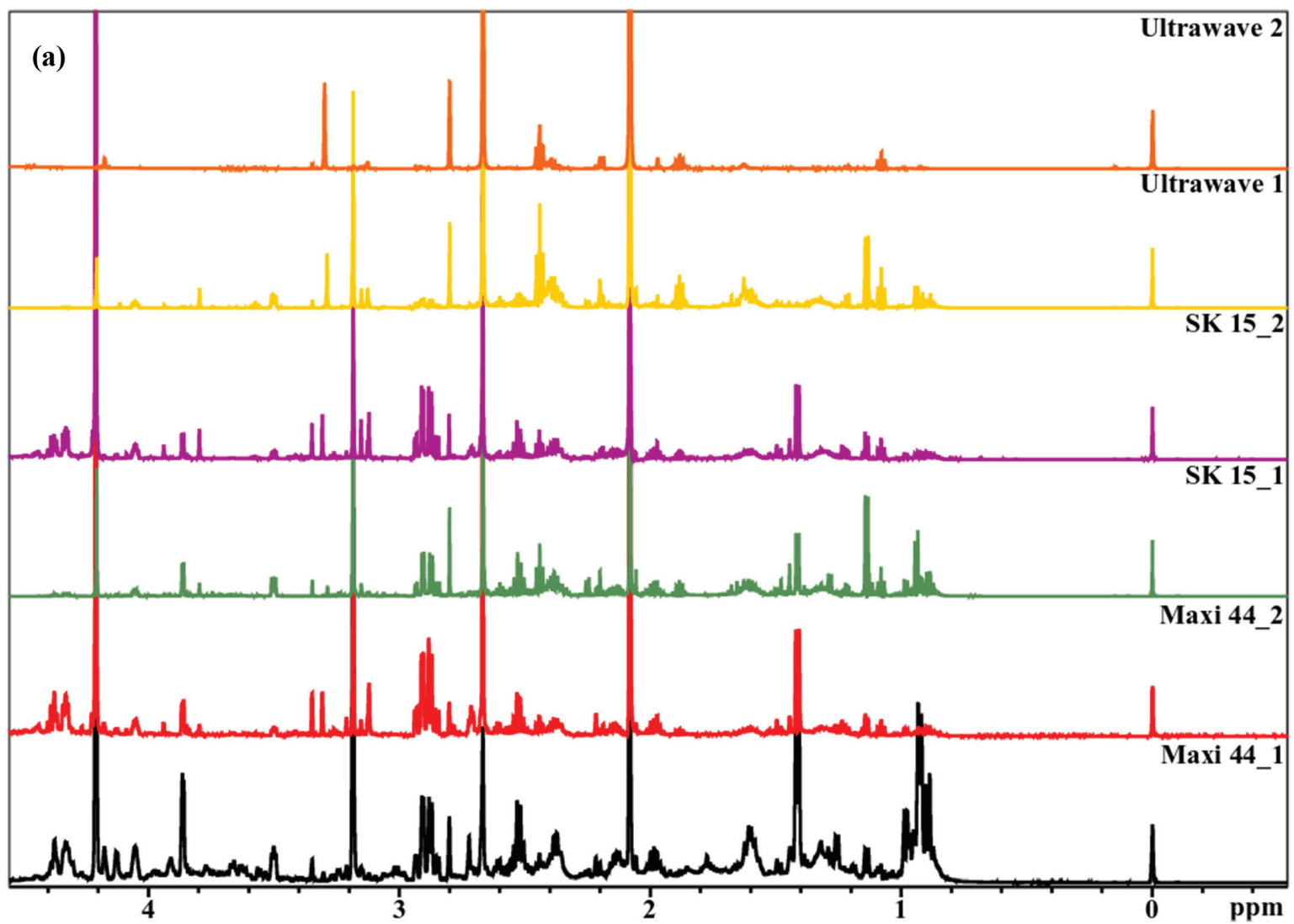

(b)

Ultrawave 2

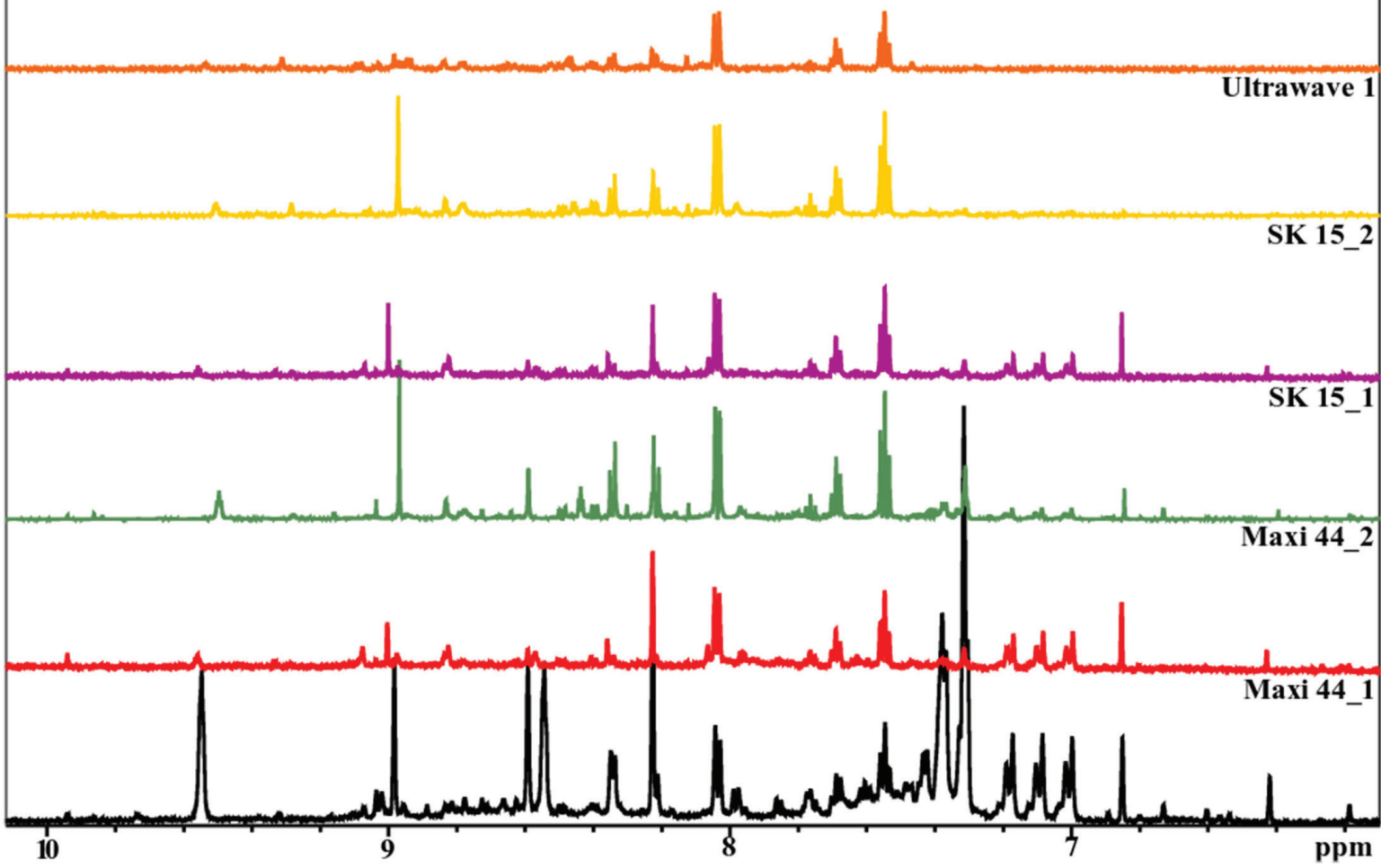

Figure 5. ${ }^{1} \mathrm{H}$ NMR spectra of organic residues in aliphatic region (a) and aromatic region (b) of digested samples in different conditions: black and red spectra (MAXI 44 without and with $\mathrm{H}_{2} \mathrm{O}_{2}$, respectively); green and purple spectra (SK-15 without and with $\mathrm{H}_{2} \mathrm{O}_{2}$, respectively); yellow and orange spectra (SRC technology at 200 and $240{ }^{\circ} \mathrm{C}$, respectively). 


\section{Acknowledgments}

The authors are grateful to the Conselho Nacional de Desenvolvimento Científico e Tecnológico (CNPq, 305201/2018-2 and 428558/2018-6) and Coordenação de Aperfeiçoamento de Pessoal de Nível Superior (CAPES/ PNPD-Graduate Program in Chemistry, Federal University of São Carlos). This study was financed in part by the Coordenação de Aperfeiçoamento de Pessoal de Nível Superior-Brasil (CAPES)-finance code 001. Instrumental support provided by Analítica (São Paulo, SP, Brazil) and Milestone (Sorisole, BG, Italy) is gratefully acknowledged. The authors also would like to express their gratitude to the Instituto Nacional de Ciências e Tecnologias Analíticas Avançadas-CNPq, grant No. 573894/2008-6 and FAPESP, grant No. 2014/50951-4.

\section{Author Contributions}

Julia A. Garitta was responsible for conceptualization, data curation, formal analysis, investigation, validation, visualization, and writing original draft; Lucimar L. Fialho for conceptualization, data curation, formal analysis, investigation, validation, visualization, and writing original draft; Glenda S. de Oliveira for formal analysis, investigation, validation, visualization, and writing original draft; Roberta M. Maria for formal analysis, investigation, validation, visualization, and writing original draft; Camillo Pirola for conceptualization, formal analysis, resources, validation, visualization, and writing-review and editing; Antônio G. Ferreira for formal analysis, validation, visualization, and writing-review and editing; Joaquim A. Nóbrega for conceptualization, data curation, formal analysis, funding acquisition, project administration, resources, validation, visualization, and writing-review and editing.

\section{References}

1. Introduction to Microwave Sample Preparation Theory and Practice, $1^{\text {st }}$ ed.; Kingston, H. M.; Jassie, L. B., eds.; ACS: Washington, USA, 1988.
2. Microwave-Enhanced Chemistry Fundamentals, Sample Preparation, and Applications, $1^{\text {st }}$ ed.; Kingston, H. M.; Haswell, S. J., eds.; ACS: Washington, USA, 1997.

3. Métodos de Preparo de Amostras para Análise Elementar, $2^{\text {nd }}$ ed.; Krug, F. J.; Rocha, F. R. P., eds.; SBQ: São Paulo, Brazil, 2019.

4. Microwave-Assisted Sample Preparation for Trace Element Determination, $1^{\text {st }}$ ed.; Flores, E. M. M., ed.; Elsevier: Amsterdam, The Netherlands, 2014.

5. Bizzi, C. A.; Pedrotti, M. F.; Silva, J. S.; Barin, J. S.; Nóbrega, J. A.; Flores, E. M. M.; J. Anal. At. Spectrom. 2017, 32, 1448.

6. Bizzi, C. A.; Flores, E. M. M.; Barin, J. S.; Garcia, E. E.; Nóbrega, J. A.; Microchem. J. 2011, 99, 193.

7. Miranda, K.; Pereira Filho, E. R.; Gomes Neto, J. A.; J. Anal. At. Spectrom. 2014, 29, 825.

8. Lautenschläger, W.; Nóbrega, J. A.; Bizzi, C. A.; Carnaroglio, D.; Colnaghi, G.; Sample Preparation for Elemental Analysis: from Evolution to Revolution. The Invention, the Technology, and the Benefits of the Single Reaction Chamber (SRC), $1^{\text {st }} \mathrm{ed}$.; Milestone: Sorisole, Italy, 2020.

9. Wieteska, E.; Zióek, A.; Drzewińska, A.; Anal. Chim. Acta 1996, $330,251$.

10. Sucharová, J.; Suchara, J.; Anal. Chim. Acta 2006, 576, 163.

11. Tavernies, I.; de Loose, M.; van Bockstaele, E.; TrAC, Trends Anal. Chem. 2004, 23, 535.

12. Bizzi, C. A.; Flores, E. M. M.; Nóbrega, J. A.; Oliveira, J. S. S.; Schmidt, L.; Mortari, S. R.; J. Anal. At. Spectrom. 2014, 29, 332.

13. Bizzi, C. A.; Barin, J. S.; Oliveira, J. S. S.; Cravotto, G.; Flores, E. M. M.; J. Braz. Chem. Soc. 2017, 28, 1673.

14. Amaral, C. D. B.; Fialho, L. L.; Camargo, F. P. R.; Pirola, C.; Nóbrega, J. A.; Talanta 2016, 160, 354.

15. Würfels, M.; Jackwerth, E.; Stoeppler, M.; Anal. Chim. Acta 1989, 226,1 .

16. Würfels, M.; Jackwerth, E.; Stoeppler, M.; Anal. Chim. Acta 1989, 226, 17. 